\title{
Inequalities on the geometric-arithmetic index
}

\author{
Ana Granados*1 (D), Ana Portilla ${ }^{1}(\mathbb{D})$, Jose M. Rodriguez ${ }^{2}(\mathbb{D})$ Jose M. Sigarreta $^{3}$ (D) \\ ${ }^{1}$ Department of Mathematics, Saint Louis University (Madrid Campus), 28003 Madrid, Spain \\ ${ }^{2}$ Departamento de Matemáticas, Universidad Carlos III de Madrid, 28911 Spain \\ ${ }^{3}$ Facultad de Matemáticas, Universidad Autónoma de Guerrero, 39650 Acalpulco Gro., Mexico
}

\begin{abstract}
Although the notion of geometric-arithmetic index has been introduced in the chemical graph theory these past years, it has already proved to be useful. The objective of the work we present here is twofold: First, obtaining new relations connecting the geometricarithmetic index with other topological indices; second, to characterize graphs which are extremal with respect to those relations.
\end{abstract}

Mathematics Subject Classification (2020). 05C07, 92E10

Keywords. geometric-arithmetic index, vertex-degree-based topological index, variable Zagreb index

\section{Introduction}

A single number (or a set of numbers) which represents a chemical structure in graphtheoretical terms via the molecular graph is called a topological descriptor; besides, if it correlates with a molecular property, it is called topological index and it is used to understand physicochemical properties of chemical compounds. The interest of topological indices lies in the fact that they synthesize some of the properties of a molecule usually into a single number, or, in some occasions, a few numbers. With this in mind, hundreds of topological indices have been introduced and studied so far; it is worth noting the seminal work by Wiener in which he used the sum of all shortest-path distances of a (molecular) graph in order to model physical properties of alkanes (see [47]).

Topological indices based on end-vertex degrees of edges have been used for 40 years and some of them are recognized tools in chemical research. Probably, the best known among such descriptors are the Randić connectivity index $(R)$ and the Zagreb indices.

The first and second Zagreb indices, denoted by $M_{1}$ and $M_{2}$ respectively, were introduced by Gutman and Trinajstić in 1972 (see [23]) as

$$
M_{1}(G)=\sum_{u \in V(G)} d_{u}^{2}, \quad M_{2}(G)=\sum_{u v \in E(G)} d_{u} d_{v}
$$

where $u v$ stands for the edge of the graph $G$ connecting vertices $u$ and $v$ and $d_{u}$ is the degree of the vertex $u$.

\footnotetext{
*Corresponding Author.

Email addresses: ana.granados@slu.edu (A. Granados), ana.portilla@slu.edu (A. Portilla), jomaro@math.uc3m.es (J. Rodriguez), jsmathguerrero@gmail.com (J. Sigarreta)

Received: 09.06.2020; Accepted: 26.12.2020
} 
Research on the Zagreb indices is abundant. In the works [19-21] and the references therein one can find details of their mathematical theory and chemical applications.

One can define the first and second variable Zagreb indices as (see e.g. [26,27,30])

$$
M_{1}^{\alpha}(G)=\sum_{u \in V(G)} d_{u}^{\alpha}, \quad M_{2}^{\alpha}(G)=\sum_{u v \in E(G)}\left(d_{u} d_{v}\right)^{\alpha}
$$

with $\alpha \in \mathbb{R}$.

Variable molecular descriptors were proposed not only as a new way of characterizing heteroatoms in molecules (see $[32,33]$ ), but also as a method to assess the structural differences. For further information see e.g. [34] and [30].

Gutman and Tošović [22] tested the correlation abilities of 20 vertex-degree-based topological indices used in the chemical literature for the case of standard heats of formation and normal boiling points of octane isomers. It is noteworthy that the second variable Zagreb index $M_{2}^{\alpha}$ with exponent $\alpha=-1$ (and to a lesser extent with exponent $\alpha=-2$ ) performs significantly better than the Randić index $\left(R=M_{2}^{-0.5}\right)$.

When it comes to the second variable Zagreb index, it has proved to be useful to model the structure-boiling point of benzenoid hydrocarbons (see [31]). Another use of variable Zagreb indices seems to be deriving multi-linear regression models [14]. Several papers discuss the relations between these indices and various properties (see, e.g., [4, 26, 28, 40, $48,49])$.

Note that $M_{1}^{2}$ is the first Zagreb index $M_{1}, M_{1}^{-1}$ is the inverse index $I D, M_{1}^{3}$ is the forgotten index $F$, etc.; also, $M_{2}^{-1 / 2}$ is the usual Randić index, $M_{2}^{1}$ is the second Zagreb index $M_{2}, M_{2}^{-1}$ is the modified Zagreb index, etc.

The general sum-connectivity index was defined by Trinajstić and Zhou in [51] as

$$
\chi_{\alpha}(G)=\sum_{u v \in E(G)}\left(d_{u}+d_{v}\right)^{\alpha} .
$$

Observe that $\chi_{1}$ coincides with the first Zagreb index $M_{1}, 2 \chi_{-1}$ with the harmonic index $H, \chi_{-1 / 2}$ with the sum-connectivity index $\chi$, etc.

The (first) geometric-arithmetic index $G A_{1}=G A$ is defined in [45] as

$$
G A_{1}(G)=G A(G)=\sum_{u v \in E(G)} \frac{\sqrt{d_{u} d_{v}}}{\frac{1}{2}\left(d_{u}+d_{v}\right)} .
$$

Although $G A$ was defined roughly ten years ago, it has been the object of study of many papers. (see, e.g., [10-12,37,45] and the references therein). In fact, it is an object highly present in current research (see e.g. $[5-7,9,18]$ and [24]). There are other geometricarithmetic indices, like $Z_{p, q}\left(Z_{0,1}=G A\right)$, but the results in [12, p.598] show that the $G A$ index gathers the same information on the molecule under study as other $Z_{p, q}$ indices.

The number of possible benzenoid hydrocarbons is huge, although only about 1,000 of them have been identified so far. As an example, there are as many as $5.85 \cdot 10^{21}$ benzenoid hydrocarbons with exactly 35 benzene rings [43]. Therefore, the ability to model their physico-chemical properties can be most helpful in order to foresee characteristics of currently unknown species. When comparing Randić index to the predicting capacity of the $G A$ index the latter is reasonably better (see [12, Table 1]). There is a good linear correlation between the heat of formation of benzenoid hydrocarbons and $G A$ as the graphic in [12, Fig.7] (from [12, Table 2], [41]) shows (with a correlation coefficient equal to 0.972 ).

Furthermore, in the case of standard enthalpy of vaporization, $G A$ index improves the performance of Randić index by more than $9 \%$. This leads to the belief that one should consider $G A$ indices when researching QSPR/QSAR.

A main topic in the study of topological indices is to find bounds of the indices involving several parameters. The objective of this paper is twofold: First, to obtain new 
relations connecting the geometric-arithmetic index with other topological indices; second, to characterize graphs which are extremal with respect to the relations obtained.

Here and hereafter, $G=(V(G), E(G))$ will denote a (non-oriented) finite simple (without multiple edges and loops) graph such that each connected component of $G$ has, at least, an edge. Also, the notation $\Delta, \delta, n, m$ for the maximum degree, the minimum degree and the cardinality of the set of vertices and edges of $G$, respectively will be used.

\section{Main results}

The following result relates the geometric-arithmetic and the first Zagreb indices.

Recall that a $(\Delta, \delta)$-biregular graph (or simply a biregular graph) is a bipartite graph for which any vertex in one side of the given bipartition has degree $\Delta$ and any vertex in the other side of the bipartition has degree $\delta$.

Theorem 2.1. If $G$ is a graph with maximum degree $\Delta$ and minimum degree $\delta$, then

$$
\begin{aligned}
G A(G) \geq \frac{1}{2 \Delta} M_{1}(G), & \text { if } \delta / \Delta \geq t_{0}, \\
G A(G) \geq \frac{2 \sqrt{\Delta \delta}}{(\Delta+\delta)^{2}} M_{1}(G), & \text { if } \delta / \Delta<t_{0},
\end{aligned}
$$

where $t_{0}$ is the unique solution of the equation $t^{3}+5 t^{2}+11 t-1=0$ in the interval $(0,1)$. The equality in the first bound is attained if and only if $G$ is regular; the equality in the second bound is attained if and only if $G$ is a biregular graph.

Proof. Since the function $h(t)=t^{3}+5 t^{2}+11 t-1$ is increasing on the interval $[0,1]$, $h(0)<0$ and $h(1)>0$, there exists a unique solution of the equation $t^{3}+5 t^{2}+11 t-1=0$ in the interval $(0,1)$. Thus, $t_{0}$ is well-defined. Since the coefficients of the polynomial $h(t)$ are rational numbers, and the coefficients of $t^{3}$ and $t^{0}$ of the polynomial $h(t)$ are 1 and -1 , respectively, we have that $t_{0} \notin \mathbb{Q}$.

We are going to compute the minimum value of the function $f:[\delta, \Delta] \times[\delta, \Delta] \rightarrow \mathbb{R}$ given by

$$
f(x, y)=\frac{2 \sqrt{x y}}{(x+y)^{2}} .
$$

By symmetry, we can assume that $x \leq y$. We have

$$
\begin{aligned}
\frac{\partial f}{\partial x}(x, y) & =\frac{x^{-1 / 2} y^{1 / 2}(x+y)^{2}-2 x^{1 / 2} y^{1 / 2} 2(x+y)}{(x+y)^{4}} \\
& =\frac{x^{-1 / 2} y^{1 / 2}(x+y)-4 x^{1 / 2} y^{1 / 2}}{(x+y)^{3}} \\
& =x^{-1 / 2} y^{1 / 2} \frac{x+y-4 x}{(x+y)^{3}} \\
& =x^{-1 / 2} y^{1 / 2} \frac{y-3 x}{(x+y)^{3}}, \\
\frac{\partial f}{\partial y}(x, y) & =y^{-1 / 2} x^{1 / 2} \frac{x-3 y}{(x+y)^{3}} .
\end{aligned}
$$

Since $y \geq x \geq \delta>0$, we have $\partial f / \partial y<0$. Thus, the minimum value of $f$ is attained on the set $\{(x, \Delta) \mid \delta \leq x \leq \Delta\}$.

If $\Delta \leq 3 \delta$, then $\partial f / \partial x(x, \Delta)<0$ for every $x>\delta$.

If $\Delta>3 \delta$, then $\partial f / \partial x(x, \Delta)>0$ for every $\delta \leq x<\Delta / 3$ and $\partial f / \partial x(x, \Delta)<0$ for every $\Delta / 3<x \leq \Delta$. 
Thus, we have in both cases

$$
f(x, y) \geq \min \{f(\Delta, \Delta), f(\delta, \Delta)\}=\min \left\{\frac{1}{2 \Delta}, \frac{2 \sqrt{\Delta \delta}}{(\Delta+\delta)^{2}}\right\} .
$$

Hence,

$$
\begin{aligned}
& \frac{2 \sqrt{d_{u} d_{v}}}{d_{u}+d_{v}} \geq \min \left\{\frac{1}{2 \Delta}, \frac{2 \sqrt{\Delta \delta}}{(\Delta+\delta)^{2}}\right\}\left(d_{u}+d_{v}\right), \\
& G A(G) \geq \min \left\{\frac{1}{2 \Delta}, \frac{2 \sqrt{\Delta \delta}}{(\Delta+\delta)^{2}}\right\} M_{1}(G) .
\end{aligned}
$$

In order to prove (2.1), it suffices to show that the inequality

$$
\frac{1}{2 \Delta} \leq \frac{2 \sqrt{\Delta \delta}}{(\Delta+\delta)^{2}}
$$

holds if and only if $\delta / \Delta \geq t_{0}$.

Inequality (2.2) is equivalent to the following statements

$$
\begin{aligned}
& (\Delta+\delta)^{2} \leq 4 \Delta \sqrt{\Delta \delta}, \quad\left(1+\frac{\delta}{\Delta}\right)^{2} \leq 4 \sqrt{\frac{\delta}{\Delta}}, \\
& \left(1+\frac{\delta}{\Delta}\right)^{4} \leq 16 \frac{\delta}{\Delta}, \quad \frac{\delta^{4}}{\Delta^{4}}+4 \frac{\delta^{3}}{\Delta^{3}}+6 \frac{\delta^{2}}{\Delta^{2}}-12 \frac{\delta}{\Delta}+1 \leq 0 .
\end{aligned}
$$

Since $0<\delta / \Delta \leq 1$, let us consider the function $g(t)=t^{4}+4 t^{3}+6 t^{2}-12 t+1$ for $t \in(0,1]$. Since $g(t)=(t-1)\left(t^{3}+5 t^{2}+11 t-1\right)=(t-1) h(t)$, we have $g(t) \leq 0$ if and only if $t \in\left[t_{0}, 1\right]$. Hence, inequality (2.2) holds if and only if $\delta / \Delta \geq t_{0}$. Note that this condition is equivalent to $\delta / \Delta>t_{0}$, since $t_{0} \notin \mathbb{Q}$; therefore, the equality in (2.2) is attained if and only if $\delta=\Delta$.

Therefore, (2.1) holds.

If $\delta / \Delta \geq t_{0}$, then the previous argument gives that $f$ attains its minimum value just at the point $(\Delta, \Delta)$. Thus, the equality in $(2.1)$ is attained if and only if $\left(d_{u}, d_{v}\right)=(\Delta, \Delta)$ for every $u v \in E(G)$, i.e., $G$ is regular.

If $\delta / \Delta<t_{0}$, then $f$ attains its minimum value just at the points $(\delta, \Delta)$ and $(\Delta, \delta)$. Therefore, the equality in (2.1) is attained if and only if $\left\{d_{u}, d_{v}\right\}=\{\delta, \Delta\}$ for every $u v \in E(G)$, i.e., $G$ is biregular.

We need the following particular case of Jensen's inequality.

Lemma 2.2. If $f$ is a convex function in an interval $I$ and $x_{1}, \ldots, x_{m} \in I$, then

$$
f\left(\frac{x_{1}+\cdots+x_{m}}{m}\right) \leq \frac{1}{m}\left(f\left(x_{1}\right)+\cdots+f\left(x_{m}\right)\right) .
$$

Theorem 2.3. If $G$ is a graph with $m$ edges, maximum degree $\Delta$ and minimum degree $\delta$, then

$$
\begin{aligned}
G A(G) \geq \frac{m^{2}}{\Delta H(G)}, & \text { if } \delta / \Delta \geq t_{0}, \\
G A(G) \geq \frac{4 \sqrt{\Delta \delta} m^{2}}{(\Delta+\delta)^{2} H(G)}, & \text { if } \delta / \Delta<t_{0},
\end{aligned}
$$

where $t_{0}$ is the unique solution of the equation $t^{3}+5 t^{2}+11 t-1=0$ in the interval $(0,1)$. The equality in the first bound is attained if and only if $G$ is regular; the equality in the second bound is attained if and only if $G$ is a biregular graph.

Proof. The argument in the proof of Theorem 2.1 gives

$$
\frac{d_{u}+d_{v}}{2 \sqrt{d_{u} d_{v}}} \leq\left(\min \left\{\frac{1}{2 \Delta}, \frac{2 \sqrt{\Delta \delta}}{(\Delta+\delta)^{2}}\right\}\right)^{-1} \frac{1}{d_{u}+d_{v}},
$$


for every $u v \in E(G)$. Since $f(x)=1 / x$ is a convex function in $\mathbb{R}_{+}$, Lemma 2.2 gives

$$
\begin{aligned}
\frac{m}{G A(G)} & =\frac{m}{\sum_{u v \in E(G) \frac{2 \sqrt{d_{u} d_{v}}}{d_{u}+d_{v}}}} \leq \frac{1}{m} \sum_{u v \in E(G)} \frac{d_{u}+d_{v}}{2 \sqrt{d_{u} d_{v}}} \\
& \leq \frac{1}{2 m}\left(\min \left\{\frac{1}{2 \Delta}, \frac{2 \sqrt{\Delta \delta}}{(\Delta+\delta)^{2}}\right\}\right)^{-1} \sum_{u v \in E(G)} \frac{2}{d_{u}+d_{v}} \\
& =\frac{1}{2 m}\left(\min \left\{\frac{1}{2 \Delta}, \frac{2 \sqrt{\Delta \delta}}{(\Delta+\delta)^{2}}\right\}\right)^{-1} H(G) .
\end{aligned}
$$

The argument in the proof of Theorem 2.1 gives

$$
\begin{aligned}
\min \left\{\frac{1}{2 \Delta}, \frac{2 \sqrt{\Delta \delta}}{(\Delta+\delta)^{2}}\right\}=\frac{1}{2 \Delta}, & \text { if } \delta / \Delta \geq t_{0}, \\
\min \left\{\frac{1}{2 \Delta}, \frac{2 \sqrt{\Delta \delta}}{(\Delta+\delta)^{2}}\right\}=\frac{2 \sqrt{\Delta \delta}}{(\Delta+\delta)^{2}}, & \text { if } \delta / \Delta<t_{0},
\end{aligned}
$$

Thus, (2.3) holds.

If the graph is regular or biregular, then we have, respectively,

$$
\begin{aligned}
\frac{m^{2}}{\Delta H(G)} & =\frac{m^{2}}{\Delta m / \Delta}=m=G A(G) \\
\frac{4 \sqrt{\Delta \delta} m^{2}}{(\Delta+\delta)^{2} H(G)} & =\frac{4 \sqrt{\Delta \delta} m^{2}}{(\Delta+\delta)^{2} 2 m /(\Delta+\delta)}=\frac{2 \sqrt{\Delta \delta}}{\Delta+\delta} m=G A(G) .
\end{aligned}
$$

If the equality in the first bound in (2.3) is attained, then the argument in the proof of Theorem 2.1 gives that $G$ is regular.

If the equality in the second bound in (2.3) is attained, then the equality in the proof of Theorem 2.1 gives that $G$ is biregular.

Theorem 2.4. If $G$ is a graph with $m$ edges and minimum degree $\delta$, then

$$
G A(G) \geq m-\frac{M_{1}(G)-2 M_{2}^{1 / 2}(G)}{2 \delta},
$$

and the equality is attained if and only if $G$ is regular.

Proof. We have

Since

$$
\begin{aligned}
& \frac{2 \sqrt{d_{u} d_{v}}}{d_{u}+d_{v}}+\frac{\left(\sqrt{d_{u}}-\sqrt{d_{v}}\right)^{2}}{d_{u}+d_{v}}=1 \\
& G A(G)+\sum_{u v \in E(G)} \frac{\left(\sqrt{d_{u}}-\sqrt{d_{v}}\right)^{2}}{d_{u}+d_{v}}=m .
\end{aligned}
$$

we conclude

$$
\begin{aligned}
& \sum_{u v \in E(G)} \frac{\left(\sqrt{d_{u}}-\sqrt{d_{v}}\right)^{2}}{d_{u}+d_{v}} \leq \frac{1}{2 \delta} \sum_{u v \in E(G)}\left(\sqrt{d_{u}}-\sqrt{d_{v}}\right)^{2} \\
& \quad=\frac{1}{2 \delta}\left(\sum_{u v \in E(G)}\left(d_{u}+d_{v}\right)-2 \sum_{u v \in E(G)} \sqrt{d_{u} d_{v}}\right)=\frac{M_{1}(G)-2 M_{2}^{1 / 2}(G)}{2 \delta}
\end{aligned}
$$

If $G$ is regular, then

$$
G A(G) \geq m-\frac{M_{1}(G)-2 M_{2}^{1 / 2}(G)}{2 \delta} .
$$

$$
m-\frac{M_{1}(G)-2 M_{2}^{1 / 2}(G)}{2 \delta}=m-\frac{2 \delta m-2 \delta m}{2 \delta}=m=G A(G) .
$$


If the equality is attained, then $d_{u}+d_{v}=2 \delta$; thus, $d_{u}=\delta$ for every $u \in V(G)$, and $G$ is a regular graph.

Remark 2.5. Since $M_{1}(G)-2 M_{2}^{1 / 2}(G)=\sum_{u v \in E(G)}\left(\sqrt{d_{u}}-\sqrt{d_{v}}\right)^{2}$, we have $M_{1}(G)-$ $2 M_{2}^{1 / 2}(G) \geq 0$.

Theorem 2.6. If $\alpha>0$ and $G$ is a graph with $m$ edges and minimum degree $\delta$, then

$$
G A(G) \geq 2 \delta m^{(\alpha+1) / \alpha} \chi_{\alpha}(G)^{-1 / \alpha},
$$

and the equality is attained if and only if $G$ is regular.

Proof. Let us define $p=(\alpha+1) / \alpha>1$. Hölder inequality gives

$$
\begin{aligned}
m & =\sum_{u v \in E(G)}\left(\frac{\sqrt{d_{u} d_{v}}}{d_{u}+d_{v}}\right)^{1 / p}\left(\frac{d_{u}+d_{v}}{\sqrt{d_{u} d_{v}}}\right)^{1 / p} \\
& \leq\left(\sum_{u v \in E(G)} \frac{\sqrt{d_{u} d_{v}}}{d_{u}+d_{v}}\right)^{1 / p}\left(\sum_{u v \in E(G)}\left(\frac{d_{u}+d_{v}}{\sqrt{d_{u} d_{v}}}\right)^{1 /(p-1)}\right)^{(p-1) / p}, \\
m^{p} & \leq \frac{1}{2} G A(G)\left(\frac{1}{\delta^{1 /(p-1)}} \chi_{1 /(p-1)}(G)\right)^{p-1}=\frac{1}{2 \delta} G A(G) \chi_{1 /(p-1)}(G)^{p-1} .
\end{aligned}
$$

The result follows from this inequality and the equality $p-1=1 / \alpha$.

If the graph is regular, then

$$
2 \delta m^{(\alpha+1) / \alpha} \chi_{\alpha}(G)^{-1 / \alpha}=2 \delta m^{1 / \alpha} m\left((2 \delta)^{\alpha} m\right)^{-1 / \alpha}=m=G A(G) .
$$

If the equality is attained, then the previous argument gives $\sqrt{d_{u} d_{v}}=\delta$ for every $u v \in E(G)$. Thus, $d_{u}=\delta$ for every $u \in V(G)$, and $G$ is a regular graph.

As a consequence of Theorem 2.6, we obtain the following result appearing in [36, Theorem 3.7].

Corollary 2.7. If $G$ is a graph with $m$ edges and minimum degree $\delta$, then

$$
G A(G) \geq \frac{2 \delta m^{2}}{M_{1}(G)}
$$

and the equality is attained if and only if $G$ is regular.

Theorem 2.8. If $p \geq 2$ and $G$ is a graph with $m$ edges and minimum degree $\delta$, then

$$
G A(G) \geq 2 \delta^{1 / 2} m^{p} M_{1}^{(2 p-1) /(2 p-2)}(G)^{1-p} .
$$

Proof. Hölder inequality gives

$$
\begin{aligned}
m & =\sum_{u v \in E(G)}\left(\frac{\sqrt{d_{u} d_{v}}}{d_{u}+d_{v}}\right)^{1 / p}\left(\frac{d_{u}+d_{v}}{\sqrt{d_{u} d_{v}}}\right)^{1 / p} \\
& \leq\left(\sum_{u v \in E(G)} \frac{\sqrt{d_{u} d_{v}}}{d_{u}+d_{v}}\right)^{1 / p}\left(\sum_{u v \in E(G)}\left(\sqrt{\frac{d_{u}}{d_{v}}}+\sqrt{\frac{d_{v}}{d_{u}}}\right)^{1 /(p-1)}\right)^{(p-1) / p} .
\end{aligned}
$$

Since $p \geq 2$, we have $0<1 /(p-1) \leq 1$ and so

$$
m^{p} \leq\left(\sum_{u v \in E(G)} \frac{\sqrt{d_{u} d_{v}}}{d_{u}+d_{v}}\right)\left(\sum_{u v \in E(G)}\left(\left(\frac{d_{u}}{d_{v}}\right)^{1 /(2 p-2)}+\left(\frac{d_{v}}{d_{u}}\right)^{1 /(2 p-2)}\right)\right)^{p-1} .
$$


Since

$$
\begin{aligned}
\sum_{u v \in E(G)}\left(\left(\frac{d_{u}}{d_{v}}\right)^{1 /(2 p-2)}+\left(\frac{d_{v}}{d_{u}}\right)^{1 /(2 p-2)}\right) & \leq \delta^{-1 /(2 p-2)} \sum_{u v \in E(G)}\left(d_{u}^{1 /(2 p-2)}+d_{v}^{1 /(2 p-2)}\right) \\
& =\delta^{-1 /(2 p-2)} \sum_{u \in V(G)} d_{u}^{1 /(2 p-2)} d_{u} \\
& =\delta^{-1 /(2 p-2)} M_{1}^{(2 p-1) /(2 p-2)}(G)
\end{aligned}
$$

we have

$$
\begin{aligned}
m^{p} & \leq \frac{1}{2} G A(G)\left(\delta^{-1 /(2 p-2)} M_{1}^{(2 p-1) /(2 p-2)}(G)\right)^{p-1} \\
& =\frac{1}{2} \delta^{-1 / 2} G A(G) M_{1}^{(2 p-1) /(2 p-2)}(G)^{p-1} .
\end{aligned}
$$

We will need the following elementary result. We include a proof for the sake of completeness.

Lemma 2.9. Let $f:[a, b] \rightarrow \mathbb{R}$ be a $C^{2}$ function such that $f^{\prime}=g h$ for some $C^{1}$ functions $g, h$ with $g \geq 0$ and $h^{\prime} \geq 0$ on $[a, b]$. Then $\max _{t \in[a, b]} f(t)=\max \{f(a), f(b)\}$.

Proof. If $h \geq 0$, then $f^{\prime} \geq 0$ and $\max _{t \in[a, b]} f(t)=f(b)=\max \{f(a), f(b)\}$.

If $h \leq 0$, then $f^{\prime} \leq 0$ and $\max _{t \in[a, b]} f(t)=f(a)=\max \{f(a), f(b)\}$.

Otherwise, $h(a)<0$ and $h(b)>0$ since $h^{\prime} \geq 0$ on $[a, b]$. Thus, there exists $t_{0} \in(a, b)$ such that $h\left(t_{0}\right)=0$. Since $h^{\prime} \geq 0$ on $[a, b]$, we have $h \leq 0$ on $\left[a, t_{0}\right]$ and $h \geq 0$ on $\left[t_{0}, b\right]$. Hence, $f$ is non-increasing on $\left[a, t_{0}\right]$ and non-decreasing on $\left[t_{0}, b\right]$, and the conclusion holds.

Theorem 2.10. Let $G$ be a graph with maximum degree $\Delta$ and minimum degree $\delta$, and $\alpha \in \mathbb{R}$. Then

$$
\begin{array}{cl}
G A(G) \geq \delta^{-2 \alpha} M_{2}^{\alpha}(G), & \text { if } \alpha \leq-1 / 2, \\
G A(G) \geq \min \left\{\delta^{-2 \alpha}, \frac{2}{(\Delta \delta)^{\alpha-1 / 2}(\Delta+\delta)}\right\} M_{2}^{\alpha}(G), & \text { if }-1 / 2<\alpha \leq 0, \\
G A(G) \geq \min \left\{\Delta^{-2 \alpha}, \frac{2}{(\Delta \delta)^{\alpha-1 / 2}(\Delta+\delta)}\right\} M_{2}^{\alpha}(G), & \text { if } 0<\alpha<1 / 2, \\
G A(G) \geq \Delta^{-2 \alpha} M_{2}^{\alpha}(G), & \text { if } \alpha \geq 1 / 2 .
\end{array}
$$

Every bound is attained for every regular graph $G$. Furthermore, the bounds in the first and fourth cases are attained if and only if $G$ is regular.

Proof. We are going to compute the maximum value of the function $f:[\delta, \Delta] \times[\delta, \Delta] \rightarrow \mathbb{R}$ given by

$$
f(x, y)=\frac{(x y)^{\alpha}}{\frac{\sqrt{x y}}{x+y}}=(x y)^{\alpha-1 / 2}(x+y)=x^{\alpha+1 / 2} y^{\alpha-1 / 2}+x^{\alpha-1 / 2} y^{\alpha+1 / 2} .
$$

If $\alpha \leq-1 / 2$, then $f$ is a decreasing function in each variable, and so, $f(x, y) \leq f(\delta, \delta)=$ $2 \delta^{2 \alpha}$ and

$$
M_{2}^{\alpha}(G) \leq \delta^{2 \alpha} G A(G) .
$$

If $\alpha \geq 1 / 2$, then $f$ is an increasing function in each variable, and so, $f(x, y) \leq f(\Delta, \Delta)=$ $2 \Delta^{2 \alpha}$ and

$$
M_{2}^{\alpha}(G) \leq \Delta^{2 \alpha} G A(G) .
$$


Let us consider the case $-1 / 2<\alpha<1 / 2$. We have

$$
\begin{aligned}
\frac{\partial f}{\partial x}(x, y) & =(\alpha+1 / 2) x^{\alpha-1 / 2} y^{\alpha-1 / 2}+(\alpha-1 / 2) x^{\alpha-3 / 2} y^{\alpha+1 / 2} \\
& =x^{\alpha-3 / 2} y^{\alpha-1 / 2}((\alpha+1 / 2) x+(\alpha-1 / 2) y), \\
\frac{\partial f}{\partial y}(x, y) & =y^{\alpha-3 / 2} x^{\alpha-1 / 2}((\alpha+1 / 2) y+(\alpha-1 / 2) x) .
\end{aligned}
$$

By symmetry, we can assume that $x \leq y$.

Assume now $-1 / 2<\alpha \leq 0$. Thus, $(\alpha+1 / 2) x+(\alpha-1 / 2) y \leq(\alpha+1 / 2) y+(\alpha-1 / 2) y=$ $2 \alpha y \leq 0$ and so, $\partial f / \partial x \leq 0$. Therefore, the maximum value of $f$ is attained on the set $\{x=\delta, \delta \leq y \leq \Delta\}$.

Since the function $(\alpha+1 / 2) y+(\alpha-1 / 2) \delta$ is increasing on $y \in[\delta, \Delta]$ and $\partial f / \partial y(\delta, y)$ and $(\alpha+1 / 2) y+(\alpha-1 / 2) \delta$ have the same sign, Lemma 2.9 gives $f(\delta, y) \leq \max \{f(\delta, \delta), f(\delta, \Delta)\}$. Thus,

$$
f(x, y) \leq \max \left\{2 \delta^{2 \alpha},(\Delta \delta)^{\alpha-1 / 2}(\Delta+\delta)\right\} .
$$

Finally, assume $0<\alpha<1 / 2$. Thus, $(\alpha+1 / 2) y+(\alpha-1 / 2) x \geq(\alpha+1 / 2) x+(\alpha-1 / 2) x=$ $2 \alpha x>0$ and so, $\partial f / \partial y>0$. Therefore, the maximum value of $f$ is attained on the set $\{y=\Delta, \delta \leq x \leq \Delta\}$.

Since the function $(\alpha+1 / 2) x+(\alpha-1 / 2) \Delta$ is increasing on $x \in[\delta, \Delta]$ and $\partial f / \partial x(x, \Delta)$ and $(\alpha+1 / 2) x+(\alpha-1 / 2) \Delta$ have the same sign, Lemma 2.9 gives $f(x, \Delta) \leq \max \{f(\delta, \Delta), f(\Delta, \Delta)\}$. Therefore,

$$
f(x, y) \leq \max \left\{2 \Delta^{2 \alpha},(\Delta \delta)^{\alpha-1 / 2}(\Delta+\delta)\right\} .
$$

This finishes the proof of the inequalities.

If the graph is regular, then

and

$$
\frac{2}{(\Delta \delta)^{\alpha-1 / 2}(\Delta+\delta)}=\delta^{-2 \alpha}=\Delta^{-2 \alpha}
$$

$$
\Delta^{-2 \alpha} M_{2}^{\alpha}(G)=\Delta^{-2 \alpha} \Delta^{2 \alpha} m=G A(G) .
$$

The properties of the function $f$ give that, in the first and fourth cases, each equality is attained if and only if either $d_{u}=d_{v}=\delta$ for every $u v \in E(G)$ or $d_{u}=d_{v}=\Delta$ for every $u v \in E(G)$, and so, $G$ is regular.

Theorem 2.10 has the following consequence for the Randić, second Zagreb and reciprocal Randić indices.

Corollary 2.11. Let $G$ be a graph with maximum degree $\Delta$ and minimum degree $\delta$. Then

$$
\begin{aligned}
& G A(G) \geq \delta R(G), \\
& G A(G) \geq \Delta^{-2} M_{2}(G), \\
& G A(G) \geq \Delta^{-1} M_{2}^{1 / 2}(G) .
\end{aligned}
$$

The equality in each bound is attained if and only if $G$ is regular.

Note that the first inequality in Corollary 2.11 appears in [39, Theorem 6].

\section{Other bounds}

The Albertson index is defined in [2] (see also [3]) as

$$
\operatorname{Alb}(G)=\sum_{u v \in E(G)}\left|d_{u}-d_{v}\right| .
$$

This index is also known as third Zagreb index (see [16]) and misbalance deg index (see [46] and [44]). This is a significant predictor of standard enthalpy of vaporisation for octane isomers (see [46]). This index is much used as a measure of non-regularity of a graph. 
Theorem 3.1. If $G$ is a graph with $m$ edges and minimum degree $\delta$, then

$$
G A(G) \geq m-\frac{1}{2 \delta} \operatorname{Alb}(G),
$$

and the equality is attained if and only if $G$ is regular.

Proof. We have seen in the proof of Theorem 2.4 that

$$
G A(G)+\sum_{u v \in E(G)} \frac{\left(\sqrt{d_{u}}-\sqrt{d_{v}}\right)^{2}}{d_{u}+d_{v}}=m .
$$

We have

$$
\begin{aligned}
\left|\sqrt{d_{u}}-\sqrt{d_{v}}\right|\left|\sqrt{d_{u}}-\sqrt{d_{v}}\right| & \leq\left|\sqrt{d_{u}}-\sqrt{d_{v}}\right|\left(\sqrt{d_{u}}+\sqrt{d_{v}}\right), \\
\sum_{u v \in E(G)}\left(\sqrt{d_{u}}-\sqrt{d_{v}}\right)^{2} & \leq \sum_{u v \in E(G)}\left|d_{u}-d_{v}\right|=\operatorname{Alb}(G) .
\end{aligned}
$$

Hence,

$$
\begin{aligned}
G A(G) & =m-\sum_{u v \in E(G)} \frac{\left(\sqrt{d_{u}}-\sqrt{d_{v}}\right)^{2}}{d_{u}+d_{v}} \\
& \geq m-\frac{1}{2 \delta} \sum_{u v \in E(G)}\left(\sqrt{d_{u}}-\sqrt{d_{v}}\right)^{2} \geq m-\frac{1}{2 \delta} \operatorname{Alb}(G) .
\end{aligned}
$$

If $G$ is regular, then $G A(G)=m$ and $A l b(G)=0$ and so, the equality is attained.

If the equality is attained, then $d_{u}+d_{v}=2 \delta$ for every $u v \in E(G)$; thus, $d_{u}=\delta$ for every $u \in V(G)$ and so, $G$ is a regular graph.

Theorem 3.2. If $G$ is a graph with maximum degree $\Delta$ and minimum degree $\delta$, then

$$
A l b(G) \leq \frac{\Delta^{2}-\delta^{2}}{2 \sqrt{\Delta \delta}} G A(G),
$$

and the equality is attained if and only if $G$ is a regular or biregular graph.

Proof. We have

$$
\begin{aligned}
\frac{\left|d_{u}-d_{v}\right|}{\frac{2 \sqrt{d_{u} d_{v}}}{d_{u}+d_{v}}} & =\frac{\left|d_{u}-d_{v}\right|\left(d_{u}+d_{v}\right)}{2 \sqrt{d_{u} d_{v}}}=\frac{\left|d_{u}^{2}-d_{v}^{2}\right|}{2 \sqrt{d_{u} d_{v}}}=\frac{1}{2}\left|\sqrt{\frac{d_{u}^{3}}{d_{v}}}-\sqrt{\frac{d_{v}^{3}}{d_{u}}}\right| \\
& \leq \frac{1}{2}\left(\sqrt{\frac{\Delta^{3}}{\delta}}-\sqrt{\frac{\delta^{3}}{\Delta}}\right)=\frac{\Delta^{2}-\delta^{2}}{2 \sqrt{\Delta \delta}}, \\
\operatorname{Alb}(G) & \leq \frac{\Delta^{2}-\delta^{2}}{2 \sqrt{\Delta \delta}} G A(G) .
\end{aligned}
$$

If $G$ is a regular or biregular graph, then

$$
\frac{\Delta^{2}-\delta^{2}}{2 \sqrt{\Delta \delta}} G A(G)=\frac{\Delta^{2}-\delta^{2}}{2 \sqrt{\Delta \delta}} \frac{2 \sqrt{\Delta \delta}}{\Delta+\delta} m=(\Delta-\delta) m=A l b(G) .
$$

If the equality is attained, then the previous argument gives that $\left\{d_{u}, d_{v}\right\}=\{\Delta, \delta\}$ for every $u v \in E(G)$; thus, $G$ is a regular or biregular graph.

The following well-known result provides a converse of Cauchy-Schwarz inequality (see, e.g., [29, Lemma 3.4]).

Lemma 3.3. If $a_{j}, b_{j} \geq 0$ and $\omega b_{j} \leq a_{j} \leq \Omega b_{j}$ for $1 \leq j \leq k$, then

$$
\left(\sum_{j=1}^{k} a_{j}^{2}\right)^{1 / 2}\left(\sum_{j=1}^{k} b_{j}^{2}\right)^{1 / 2} \leq \frac{1}{2}\left(\sqrt{\frac{\Omega}{\omega}}+\sqrt{\frac{\omega}{\Omega}}\right) \sum_{j=1}^{k} a_{j} b_{j} .
$$


If $a_{j}>0$ for some $1 \leq j \leq k$, then the equality holds if and only if $\omega=\Omega$ and $a_{j}=\omega b_{j}$ for every $1 \leq j \leq k$.

Multiplicative versions of the first and the second Zagreb indices, $\Pi_{1}$ and $\Pi_{2}$, were first considered in [42], defined as

$$
\Pi_{1}(G)=\prod_{u \in V(G)} d_{u}^{2}, \quad \Pi_{2}(G)=\prod_{u v \in E(G)} d_{u} d_{v} .
$$

Also, the multiplicative sum-Zagreb index $\Pi_{1}^{*}$ was introduced in [15] as

$$
\Pi_{1}^{*}(G)=\prod_{u v \in E(G)}\left(d_{u}+d_{v}\right) .
$$

Theorem 3.4. If $G$ is a graph with $m$ edges, maximum degree $\Delta$ and minimum degree $\delta$, then

$$
G A(G) \geq \frac{m}{\Delta} \Pi_{1}(G)^{\delta /(4 m)}
$$

and the equality is attained if and only if $G$ is regular.

Proof. The multiplicative version of the first Zagreb index satisfies

$$
\Pi_{1}(G)=\prod_{u \in V(G)} d_{u}^{2}=\prod_{u v \in E(G)} d_{u}^{2 / d_{u}} d_{v}^{2 / d_{v}} \leq\left(\prod_{u v \in E(G)} d_{u} d_{v}\right)^{2 / \delta} .
$$

Using the fact that the geometric mean is at most the arithmetic mean, we obtain

$$
\begin{aligned}
\frac{1}{m} G A(G) & =\frac{1}{m} \sum_{u v \in E(G)} \frac{2 \sqrt{d_{u} d_{v}}}{d_{u}+d_{v}} \geq\left(\prod_{u v \in E(G)} \frac{2 \sqrt{d_{u} d_{v}}}{d_{u}+d_{v}}\right)^{1 / m} \\
& \geq\left(\frac{1}{\Delta^{m}} \prod_{u v \in E(G)} \sqrt{d_{u} d_{v}}\right)^{1 / m}=\frac{1}{\Delta}\left(\left(\prod_{u v \in E(G)} d_{u} d_{v}\right)^{2 / \delta}\right)^{\delta /(4 m)} \\
& \geq \frac{1}{\Delta} \Pi_{1}(G)^{\delta /(4 m)} .
\end{aligned}
$$

If the equality holds, then $d_{u}+d_{v}=2 \Delta$ for every $u v \in E(G)$; hence, $d_{u}=\Delta$ for every $u \in V(G)$ and the graph is regular.

If the graph is regular, then

$$
\frac{m}{\Delta} \Pi_{1}(G)^{\delta /(4 m)}=\frac{m}{\Delta}\left(\prod_{u v \in E(G)} \Delta^{2 / \Delta} \Delta^{2 / \Delta}\right)^{\Delta /(4 m)}=m=G A(G) .
$$

Theorem 3.5. If $\alpha>0$ and $G$ is a graph with $m$ edges and maximum degree $\Delta$, then

$$
G A(G) \geq \frac{m^{(2 \alpha+1) /(2 \alpha)}}{\Delta M_{2}^{-\alpha}(G)^{1 /(2 \alpha)}}
$$

and the equality is attained for some $\alpha$ if and only if $G$ is regular.

Proof. Using the fact that the geometric mean is at most the arithmetic mean, we obtain

$$
\begin{aligned}
\frac{1}{m} G A(G) & =\frac{1}{m} \sum_{u v \in E(G)} \frac{2 \sqrt{d_{u} d_{v}}}{d_{u}+d_{v}} \geq\left(\prod_{u v \in E(G)} \frac{2 \sqrt{d_{u} d_{v}}}{d_{u}+d_{v}}\right)^{1 / m} \\
& \geq\left(\frac{1}{\Delta^{m}} \prod_{u v \in E(G)} \sqrt{d_{u} d_{v}}\right)^{1 / m}, \\
G A(G)^{2 \alpha} & \geq\left(\frac{m}{\Delta}\right)^{2 \alpha}\left(\prod_{u v \in E(G)}\left(d_{u} d_{v}\right)^{\alpha}\right)^{1 / m} .
\end{aligned}
$$


Using the fact that the harmonic mean is at most the geometric mean, we deduce

$$
\begin{aligned}
G A(G)^{2 \alpha} & \geq\left(\frac{m}{\Delta}\right)^{2 \alpha}\left(\prod_{u v \in E(G)}\left(d_{u} d_{v}\right)^{\alpha}\right)^{1 / m} \\
& \geq\left(\frac{m}{\Delta}\right)^{2 \alpha} \frac{m}{\sum_{u v \in E(G)}\left(d_{u} d_{v}\right)^{-\alpha}}=\left(\frac{m}{\Delta}\right)^{2 \alpha} \frac{m}{M_{2}^{-\alpha}(G)} .
\end{aligned}
$$

If the equality holds, then $d_{u}+d_{v}=2 \Delta$ for every $u v \in E(G)$; hence, $d_{u}=\Delta$ for every $u \in V(G)$ and the graph is regular.

If the graph is regular, then

$$
\frac{m^{(2 \alpha+1) /(2 \alpha)}}{\Delta M_{2}^{-\alpha}(G)^{1 /(2 \alpha)}}=\frac{m^{1 /(2 \alpha)} m}{\Delta\left(\Delta^{-2 \alpha} m\right)^{1 /(2 \alpha)}}=m=G A(G) .
$$

We need the following result in [35, Theorem 3.3].

Lemma 3.6. If $G$ is a graph with maximum degree $\Delta$ and minimum degree $\delta$, then

$$
\begin{array}{ll}
\frac{\delta^{\alpha}}{2} M_{1}^{\alpha+1}(G) \leq M_{2}^{\alpha}(G) \leq \frac{\Delta^{\alpha}}{2} M_{1}^{\alpha+1}(G) & \text { if } \alpha>0, \\
\frac{\Delta^{\alpha}}{2} M_{1}^{\alpha+1}(G) \leq M_{2}^{\alpha}(G) \leq \frac{\delta^{\alpha}}{2} M_{1}^{\alpha+1}(G) & \text { if } \alpha<0,
\end{array}
$$

and the equality holds for some $\alpha$ if and only if $G$ is regular.

Corollary 3.7. If $\alpha>0$ and $G$ is a graph with $m$ edges, maximum degree $\Delta$ and minimum degree $\delta$, then

$$
G A(G) \geq \frac{2^{1 /(2 \alpha)} \delta^{1 / 2} m^{(2 \alpha+1) /(2 \alpha)}}{\Delta M_{1}^{1-\alpha}(G)^{1 /(2 \alpha)}},
$$

and the equality holds for some $\alpha$ if and only if $G$ is regular.

Proof. Theorem 3.5 and Lemma 3.6 give

$$
G A(G) \geq \frac{m^{(2 \alpha+1) /(2 \alpha)}}{\Delta M_{2}^{-\alpha}(G)^{1 /(2 \alpha)}} \geq \frac{m^{(2 \alpha+1) /(2 \alpha)}}{\Delta\left(\frac{1}{2} \delta^{-\alpha} M_{1}^{1-\alpha}(G)\right)^{1 /(2 \alpha)}}=\frac{2^{1 /(2 \alpha)} \delta^{1 / 2} m^{(2 \alpha+1) /(2 \alpha)}}{\Delta M_{1}^{1-\alpha}(G)^{1 /(2 \alpha)}} .
$$

If the graph is regular, then

$$
\frac{2^{1 /(2 \alpha)} \delta^{1 / 2} m^{(2 \alpha+1) /(2 \alpha)}}{\Delta M_{1}^{1-\alpha}(G)^{1 /(2 \alpha)}}=\frac{2^{1 /(2 \alpha)} \delta^{1 / 2} m^{1 /(2 \alpha)} m}{\delta\left(\delta^{-\alpha} \delta n\right)^{1 /(2 \alpha)}}=\frac{2^{1 /(2 \alpha)} \delta^{1 / 2} m^{1 /(2 \alpha)} m}{\delta \delta^{-1 / 2}(2 m)^{1 /(2 \alpha)}}=m=G A(G) .
$$

If the equality holds, then we have the equality in Theorem 3.5, and thus the graph is regular.

Theorem 3.8. If $G$ is a graph, then

$$
G A(G) \geq \frac{2 M_{2}^{1 / 4}(G)^{2}}{M_{1}(G)}
$$

and the equality is attained if $G$ is a regular or biregular graph.

Proof. Cauchy-Schwarz inequality gives

$$
\begin{aligned}
M_{2}^{1 / 4}(G)^{2} & =\left(\sum_{u v \in E(G)} \frac{\left(d_{u} d_{v}\right)^{1 / 4}}{\left(d_{u}+d_{v}\right)^{1 / 2}}\left(d_{u}+d_{v}\right)^{1 / 2}\right)^{2} \leq\left(\sum_{u v \in E(G)} \frac{\sqrt{d_{u} d_{v}}}{d_{u}+d_{v}}\right)\left(\sum_{u v \in E(G)}\left(d_{u}+d_{v}\right)\right) \\
& =\frac{1}{2} G A(G) M_{1}(G) .
\end{aligned}
$$


If $G$ is regular or biregular, then

$$
\frac{2 M_{2}^{1 / 4}(G)^{2}}{M_{1}(G)}=\frac{2\left((\Delta \delta)^{1 / 4} m\right)^{2}}{(\Delta+\delta) m}=\frac{2 \sqrt{\Delta \delta}}{\Delta+\delta} m=G A(G)
$$

The following technical result appears in [36, Corollary 2.3].

Lemma 3.9. Let $g$ be the function $g(x, y)=\frac{2 \sqrt{x y}}{x+y}$ with $0<a \leq x, y \leq b$. Then

$$
\frac{2 \sqrt{a b}}{a+b} \leq g(x, y) \leq 1 .
$$

The equality in the lower bound is attained if and only if either $x=a$ and $y=b$, or $x=b$ and $y=a$, and the equality in the upper bound is attained if and only if $x=y$. Besides, $g(x, y)=g\left(x^{\prime}, y^{\prime}\right)$ if and only if $x / y$ is equal to either $x^{\prime} / y^{\prime}$ or $y^{\prime} / x^{\prime}$.

As a consequence of Lemma 3.9 we obtain the following bounds for the geometricarithmetic index [10] (see also [12, p.609-610]).

Proposition 3.10. If $G$ is a graph with $m$ edges, maximum degree $\Delta$ and minimum degree $\delta$, then

$$
\frac{2 m \sqrt{\Delta \delta}}{\Delta+\delta} \leq G A(G) \leq m
$$

The equality in the first inequality is attained if and only if $G$ is regular or biregular. The equality in the second inequality is attained if and only if $G$ is regular.

The forgotten topological index (or F-index) was introduced in [23], at the same time as Zagreb indices. There, it was defined as

$$
F(G)=\sum_{u \in V(G)} d_{u}^{3}
$$

As the first Zagreb index it was used when finding the total $\pi$-electron energy (see [23]). It was thought to assess the extent of the branching of the carbon-atom skeleton of the associated molecule. Recently, when Furtula and Gutman ([17]) showed that the acetic factor and the entropy could be predicted similarly by either the $F$-index or the first Zagreb index, attention was finally given to the former. Both of them yield correlation coefficients greater than 0.95. Besides, [17] pointed out that the F-index could be employed to predict the logarithm of the octanol-water partition coefficient (see also [1]) very accuarately. For more bounds of the $F$-index see, e.g., $[1,8,38]$.

We report a mistake in the lower bound of $G A$ in [39, Theorem 5]. By using the argument in the proof of that theorem, we obtain the following inequality involving the forgotten topological index.

Theorem 3.11. If $G$ is a graph with maximum degree $\Delta$ and minimum degree $\delta$, then

$$
G A(G) \geq \frac{4 \Delta^{2} \delta^{2} \sqrt{\left(F(G)+2 M_{2}(G)\right) M_{2}^{-1}(G)}}{(\Delta+\delta)^{2}\left(\Delta^{2}+\delta^{2}\right)},
$$

and the equality is attained if and only if $G$ is regular.

Proof. We have by Lemma 3.9

$$
\frac{4 \Delta \delta}{(\Delta+\delta)^{2}} \leq \frac{4 d_{u} d_{v}}{\left(d_{u}+d_{v}\right)^{2}} \leq 1
$$


for every $u v \in E(G)$. Hence,

$$
\frac{2 \sqrt{d_{u} d_{v}}}{d_{u}+d_{v}} \geq \frac{4 \Delta \delta}{(\Delta+\delta)^{2}} \frac{d_{u}+d_{v}}{2 \sqrt{d_{u} d_{v}}}, \quad G A(G) \geq \frac{4 \Delta \delta}{(\Delta+\delta)^{2}} \sum_{u v \in E(G)} \frac{d_{u}+d_{v}}{2 \sqrt{d_{u} d_{v}}} .
$$

Moreover,

Since

$$
\begin{aligned}
\sum_{u v \in E(G)}\left(d_{u}+d_{v}\right)^{2} & =\sum_{u v \in E(G)}\left(d_{u}^{2}+d_{v}^{2}\right)+2 \sum_{u v \in E(G)} d_{u} d_{v} \\
& =\sum_{u \in V(G)} d_{u}^{3}+2 M_{2}(G) \\
& =F(G)+2 M_{2}(G) .
\end{aligned}
$$

$$
\delta^{2} \leq \frac{\left(d_{u}+d_{v}\right) / 2}{1 / \sqrt{d_{u} d_{v}}}=\frac{d_{u}+d_{v}}{2} \sqrt{d_{u} d_{v}} \leq \Delta^{2}
$$

Lemma 3.3 gives

$$
\begin{aligned}
\sum_{u v \in E(G)} \frac{d_{u}+d_{v}}{2 \sqrt{d_{u} d_{v}}} & \geq \frac{\left(\sum_{u v \in E(G)} \frac{\left(d_{u}+d_{v}\right)^{2}}{4}\right)^{1 / 2}\left(\sum_{u v \in E(G)} \frac{1}{d_{u} d_{v}}\right)^{1 / 2}}{\frac{1}{2}\left(\frac{\Delta}{\delta}+\frac{\delta}{\Delta}\right)} \\
& =\frac{\Delta \delta \sqrt{\left(F(G)+2 M_{2}(G)\right) M_{2}^{-1}(G)}}{\Delta^{2}+\delta^{2}}
\end{aligned}
$$

and the desired inequality follows.

If the graph is regular, then

$$
\frac{4 \Delta^{2} \delta^{2} \sqrt{\left(F(G)+2 M_{2}(G)\right) M_{2}^{-1}(G)}}{(\Delta+\delta)^{2}\left(\Delta^{2}+\delta^{2}\right)}=\frac{\sqrt{\left(2 \Delta^{2} m+2 \Delta^{2} m\right) m / \Delta^{2}}}{2}=m=G A(G) .
$$

If the equality is attained, then Lemma 3.3 gives $\delta^{2}=\Delta^{2}$ and $G$ is a regular graph.

Theorem 3.12. If $G$ is a graph with maximum degree $\Delta$ and minimum degree $\delta$, then

$$
G A(G) \geq \frac{F(G)}{2 \Delta^{2}}-\frac{(\Delta-\delta) A l b(G)}{2 \Delta \delta},
$$

and the equality is attained if and only if $G$ is regular.

Proof. Since

$$
\frac{d_{u}^{2}+d_{v}^{2}}{2 \Delta} \leq \frac{d_{u}^{2}+d_{v}^{2}}{d_{u}+d_{v}}=\frac{2 d_{u} d_{v}}{d_{u}+d_{v}}+\frac{\left(d_{u}-d_{v}\right)^{2}}{d_{u}+d_{v}} \leq \frac{2 \Delta \sqrt{d_{u} d_{v}}}{d_{u}+d_{v}}+\frac{\left(d_{u}-d_{v}\right)^{2}}{d_{u}+d_{v}},
$$

for every $u v \in E(G)$, we have

$$
\frac{F(G)}{2 \Delta} \leq \Delta G A(G)+\sum_{u v \in E(G)} \frac{\left(d_{u}-d_{v}\right)^{2}}{d_{u}+d_{v}}
$$

Since

$$
\sum_{u v \in E(G)} \frac{\left(d_{u}-d_{v}\right)^{2}}{d_{u}+d_{v}} \leq \frac{\Delta-\delta}{2 \delta} \sum_{u v \in E(G)}\left|d_{u}-d_{v}\right|=\frac{\Delta-\delta}{2 \delta} A l b(G),
$$

we conclude

$$
\frac{F(G)}{2 \Delta} \leq \Delta G A(G)+\frac{(\Delta-\delta) A l b(G)}{2 \delta} .
$$

If the graph is regular, then

$$
\frac{F(G)}{2 \Delta^{2}}-\frac{(\Delta-\delta) A l b(G)}{2 \Delta \delta}=\frac{F(G)}{2 \Delta^{2}}=\frac{2 \Delta^{2} m}{2 \Delta^{2}}=m=G A(G) .
$$


The previous argument gives that if the bound is attained, then $d_{u}+d_{v}=2 \Delta$ for every $u v \in E(G)$. Thus, $d_{u}=\Delta$ for every $u \in V(G)$ and $G$ is regular.

The following Kober's inequality appears in [25] (see also [50, Lemma 1]).

Lemma 3.13. If $a_{j}>0$ for $1 \leq j \leq k$, then

$$
\left(\sum_{j=1}^{k} \sqrt{a_{j}}\right)^{2} \geq \sum_{j=1}^{k} a_{j}+k(k-1)\left(\prod_{j=1}^{k} a_{j}\right)^{1 / k} .
$$

A family of degree-based structure-descriptors, named Adriatic indices, was put forward in $[44,46]$. Twenty of them were selected as significant predictors. One of them, the inverse sum indeg index, ISI, was singled out in [44] as a significant predictor of total surface area of octane isomers. This index is defined as

$$
I S I(G)=\sum_{u v \in E(G)} \frac{d_{u} d_{v}}{d_{u}+d_{v}}=\sum_{u v \in E(G)} \frac{1}{\frac{1}{d_{u}}+\frac{1}{d_{v}}} .
$$

In the last years there is an increasing interest in the mathematical properties of this index.

Theorem 3.14. If $G$ is a graph with $m$ edges and maximum degree $\Delta$, then

$$
G A(G) \geq \sqrt{\frac{2}{\Delta}\left(I S I(G)+m(m-1) \frac{\Pi_{2}(G)^{1 / m}}{\Pi_{1}^{*}(G)^{1 / m}}\right)},
$$

and the equality is attained if and only if $G$ is a regular graph.

Proof. Lemma 3.13 gives

$$
\begin{aligned}
& I S I(G)+m(m-1) \frac{\Pi_{2}(G)^{1 / m}}{\Pi_{1}^{*}(G)^{1 / m}} \\
& =\sum_{u v \in E(G)} \frac{d_{u} d_{v}}{d_{u}+d_{v}}+m(m-1)\left(\prod_{u v \in E(G)} \frac{d_{u} d_{v}}{d_{u}+d_{v}}\right)^{1 / m} \leq\left(\sum_{u v \in E(G)} \frac{\left(d_{u} d_{v}\right)^{1 / 2}}{\left(d_{u}+d_{v}\right)^{1 / 2}}\right)^{2} \\
& =\left(\frac{1}{\sqrt{2}} \sum_{u v \in E(G)} \frac{2 \sqrt{d_{u} d_{v}}}{d_{u}+d_{v}}\left(\frac{d_{u}+d_{v}}{2}\right)^{1 / 2}\right)^{2} \leq \frac{\Delta}{2} G A(G)^{2} .
\end{aligned}
$$

If $G$ is regular, then

$$
\begin{aligned}
& \sqrt{\frac{2}{\Delta}\left(I S I(G)+m(m-1) \frac{\Pi_{2}(G)^{1 / m}}{\Pi_{1}^{*}(G)^{1 / m}}\right)} \\
& \quad=\sqrt{\frac{2}{\Delta}\left(\frac{\Delta m}{2}+m(m-1) \frac{\left(\Delta^{2 m}\right)^{1 / m}}{\left((2 \Delta)^{m}\right)^{1 / m}}\right)}=m=G A(G) .
\end{aligned}
$$

If the equality is attained, then the previous argument gives $d_{u}+d_{v}=2 \Delta$ for every $u v \in E(G)$, i.e., $d_{u}=\Delta$ for every $u \in V(G)$ and so, $G$ is a regular graph.

We need the following Diaz-Metcalf inequality [13].

Lemma 3.15. If $a_{j}$ and $b_{j}$ are real numbers such that $h a_{j} \leq b_{j} \leq H a_{j}$ for $1 \leq j \leq m$, then

$$
\sum_{j=1}^{m} b_{j}^{2}+h H \sum_{j=1}^{m} a_{j}^{2} \leq(h+H) \sum_{j=1}^{m} a_{j} b_{j},
$$

where equality holds if and only if $b_{j}=h a_{j}$ or $b_{j}=H a_{j}$ for $1 \leq j \leq m$. 
Theorem 3.16. If $G$ is a graph with maximum degree $\Delta$ and minimum degree $\delta$, then

$$
G A(G) \geq \frac{M_{2}(G)+4 \Delta^{2} \delta^{2} \chi_{-2}(G)}{\Delta^{2}+\delta^{2}}
$$

and the equality is attained if and only if each connected component of $G$ is $\delta$-regular or $\Delta$-regular. In particular, if $G$ is connected, then the equality is attained if and only if $G$ is regular.

Proof. If we define

$$
a_{j}=\frac{1}{d_{u}+d_{v}}, \quad b_{j}=2 \sqrt{d_{u} d_{v}},
$$

then

$$
4 \delta^{2} \leq b_{j} / a_{j}=2 \sqrt{d_{u} d_{v}}\left(d_{u}+d_{v}\right) \leq 4 \Delta^{2},
$$

and so, Lemma 3.15 gives

$$
\begin{gathered}
4 \sum_{u v \in E(G)} d_{u} d_{v}+16 \Delta^{2} \delta^{2} \sum_{u v \in E(G)} \frac{1}{\left(d_{u}+d_{v}\right)^{2}} \leq\left(4 \Delta^{2}+4 \delta^{2}\right) \sum_{u v \in E(G)} \frac{2 \sqrt{d_{u} d_{v}}}{d_{u}+d_{v}} \\
M_{2}(G)+4 \Delta^{2} \delta^{2} \chi_{-2}(G) \leq\left(\Delta^{2}+\delta^{2}\right) G A(G) .
\end{gathered}
$$

By Lemma 3.15, the equality is attained if and only if $2 \sqrt{d_{u} d_{v}}\left(d_{u}+d_{v}\right)=4 \delta^{2}$ or $2 \sqrt{d_{u} d_{v}}\left(d_{u}+d_{v}\right)=4 \Delta^{2}$ for each $u v \in E(G)$, and this is equivalent to $d_{u}=d_{v}=\delta$ or $d_{u}=d_{v}=\Delta$ for each $u v \in E(G)$, i.e., each connected component of $G$ is $\delta$-regular or $\Delta$-regular.

Acknowledgment. All authors are supported in part by two grants from Ministerio de Economía y Competitividad, Agencia Estatal de InvestigaciÛn (AEI) and Fondo Europeo de Desarrollo Regional (FEDER) (MTM2016-78227-C2-1-P and MTM2017-90584-REDT), Spain.

\section{References}

[1] H. Abdo, D. Dimitrov and I. Gutman, On extremal trees with respect to the F-index, Kuwait J. Sci. 44 (3) 1-8, 2017.

[2] M.O. Albertson, The irregularity of a graph, Ars Comb. 46, 219-225, 1997.

[3] A. Ali, I. Gutman, E. Milovanović and I. Milovanović, Sum of Powers of the Degrees of Graphs: Extremal Results and Bounds, MATCH Commun. Math. Comput. Chem. 80, 5-84, 2018.

[4] V. Andova and M. Petrusevski, Variable Zagreb Indices and Karamataís Inequality, MATCH Commun. Math. Comput. Chem. 65, 685-690, 2011.

[5] M. Aouchiche and P. Hansen, Comparing the geometric-arithmetic Index and the spectral radius of graphs, MATCH Commun. Math. Comput. Chem. 84 (2), 473-482, 2020.

[6] M. Aouchiche and V. Ganesan, Adjusting geometric-arithmetic index to estimate boiling point, MATCH Commun. Math. Comput. Chem. 84, 483-497, 2020.

[7] M. Aouchiche, I. El Hallaoui and P. Hansen, Geometric-Arithmetic index and minimum degree of connected graphs, MATCH Commun. Math. Comput. Chem. 83 (1), 179-188, 2020.

[8] Z. Che and Z. Chen, Lower and Upper Bounds of the Forgotten Topological Index, MATCH Commun. Math. Comput. Chem. 76, 635-648, 2016.

[9] Y. Chen and B. Wu On the geometric-arithmetic index of a graph, Discrete Appl. Math. 254, 268-273, 2019.

[10] K.C. Das, On geometric-arithmetic index of graphs, MATCH Commun. Math. Comput. Chem. 64, 619-630, 2010. 
[11] K.C. Das, I. Gutman and B. Furtula, On first geometric-arithmetic index of graphs, Discrete Appl. Math. 159, 2030-2037, 2011.

[12] K.C. Das, I. Gutman and B. Furtula, Survey on Geometric-Arithmetic Indices of Graphs, MATCH Commun. Math. Comput. Chem. 65, 595-644, 2011.

[13] S.S. Dragomir, A Survey On Cauchy-Bunyakovosky-Schwarz Type Discrete Inequalities, J. Inequal. Pure and Appl. Math. 4 (3), Art. 63, 2003.

[14] M. Drmota, Random Trees: An Interplay Between Combinatorics and Probability, Springer, Wien-New York, 2009.

[15] M. Eliasi, A. Iranmanesh and I. Gutman, Multiplicative versions of first Zagreb index, Commun. Math. Comput. Chem. 68 (1), 217-230, 2012.

[16] G.H. Fath-Tabar, Old and New Zagreb Indices of Graphs, MATCH Commun. Math. Comput. Chem. 65, 79-84, 2011.

[17] B. Furtula and I. Gutman, A forgotten topological index, J. Math. Chem. 53 (4), 1184-1190, 2015.

[18] X. Guo and Y. Gao Arithmetic-geometric spectral radius and energy of graphs, MATCH Commun. Math. Comput. Chem. 83, 651-660, 2020.

[19] I. Gutman, Degree-based topological indices, Croat. Chem. Acta 86, 351-361, 2013.

[20] I. Gutman and K.C. Das, The first Zagreb index 30 years after, MATCH Commun. Math. Comput. Chem. 50, 83-92, 2004.

[21] I. Gutman and T. Réti, Zagreb group indices and beyond, Int. J. Chem. Model. 6 (2-3), 191-200, 2014.

[22] I. Gutman and J. Tošović, Testing the quality of molecular structure descriptors. Vertex-degreebased topological indices, J. Serb. Chem. Soc. 78 (6), 805-810, 2013.

[23] I. Gutman and N. Trinajstić, Graph theory and molecular orbitals. Total $\pi$-electron energy of alternant hydrocarbons, Chem. Phys. Lett. 17, 535-538, 1972.

[24] J.C. Hernandez, J.M. Rodriguez and J.M. Sigarreta On the geometric-arithmetic index by decompositions, J. Math. Chem. 55, 1376-1391, 2017.

[25] H. Kober, On the arithmetic and geometric means and on Hölderís inequality, Proc. Amer. Math. Soc. 9, 452-459, 1958.

[26] X. Li and H. Zhao, Trees with the first smallest and largest generalized topological indices, MATCH Commun. Math. Comput. Chem. 50, 57-62, 2004.

[27] X. Li and J. Zheng, A unified approach to the extremal trees for different indices, MATCH Commun. Math. Comput. Chem. 54, 195-208, 2005.

[28] M. Liu and B. Liu, Some properties of the first general Zagreb index, Australas. J. Combin. 47, 285-294, 2010.

[29] A. Martínez-Pérez, J.M. Rodríguez and J.M. Sigarreta, A new approximation to the geometric-arithmetic index, J. Math. Chem. 56, 1865-1883, 2018, DOI: 10.1007/s10910-017-0811-3.

[30] A. Miličević and S. Nikolić, On variable Zagreb indices, Croat. Chem. Acta 77, 97$101,2004$.

[31] S. Nikolić, A. Miličević, N. Trinajstić and A. Jurić, On Use of the Variable Zagreb ${ }^{\nu} M_{2}$ Index in QSPR: Boiling Points of Benzenoid Hydrocarbons, Molecules 9, 1208-1221, 2004.

[32] M. Randić, Novel graph theoretical approach to heteroatoms in QSAR, Chemometrics Intel. Lab. Syst. 10, 213-227, 1991.

[33] M. Randić, On computation of optimal parameters for multivariate analysis of structure-property relationship, J. Chem. Inf. Comput. Sci. 31, 970-980, 1991.

[34] M. Randić, D. Plavšić and N. Lerš, Variable connectivity index for cycle-containing structures, J. Chem. Inf. Comput. Sci. 41, 657-662, 2001.

[35] J.M. Rodríguez, J.L. Sánchez and J.M. Sigarreta, On the first general Zagreb index, J. Math. Chem. 56, 1849-1864, 2018, DOI: 10.1007/s10910-017-0816-y. 
[36] J.M. Rodríguez and J.M. Sigarreta, On the Geometric-Arithmetic Index, MATCH Commun. Math. Comput. Chem. 74, 103-120, 2015.

[37] J.M. Rodríguez and J.M. Sigarreta, Spectral properties of geometric-arithmetic index, Appl. Math. Comput. 277, 142-153, 2016.

[38] J.M. Rodríguez and J.M. Sigarreta, New Results on the Harmonic Index and Its Generalizations, MATCH Commun. Math. Comput. Chem. 78 (2), 387-404, 2017.

[39] J.M. Sigarreta, Bounds for the geometric-arithmetic index of a graph, Miskolc Math. Notes, 16 (2), 1199-1212, 2015.

[40] M. Singh, K.Ch. Das, S. Gupta and A.K. Madan, Refined variable Zagreb indices: highly discriminating topological descriptors for QSAR/QSPR, Int. J. Chem. Modeling, 6 (2-3), 403-428, 2014.

[41] TRC Thermodynamic Tables. Hydrocarbons; Thermodynamic Research Center, The Texas A \& M University System: College Station, TX, 1987.

[42] R. Todeschini and V. Consonni, New local vertex invariants and molecular descriptors based on functions of the vertex degrees, MATCH Commun. Math. Comput. Chem. 64 (2), 359-372, 2010.

[43] M. Vöge, A.J. Guttmann and I. Jensen, On the number of benzenoid hydrocarbons, J. Chem. Inf. Comput. Sci. 42, 456-466, 2002.

[44] D. Vukičević, Bond additive modeling 2. Mathematical properties of max-min rodeg index, Croat. Chem. Acta, 83, 261-273, 2010.

[45] D. Vukičević and B. Furtula, Topological index based on the ratios of geometrical and arithmetical means of end-vertex degrees of edges, J. Math. Chem. 46, 1369-1376, 2009.

[46] D. Vukičević and M. Gašperov, Bond Additive Modeling 1. Adriatic Indices, Croat. Chem. Acta, 83 (3), 243-260, 2010.

[47] H. Wiener, Structural determination of paraffin boiling points, J. Am. Chem. Soc. 69, 17-20, 1947.

[48] S. Zhang, W. Wang and T.C.E. Cheng, Bicyclic graphs with the first three smallest and largest values of the first general Zagreb index, MATCH Commun. Math. Comput. Chem. 55, 579-592, 2006.

[49] H. Zhang and S. Zhang, Unicyclic graphs with the first three smallest and largest values of the first general Zagreb index, MATCH Commun. Math. Comput. Chem. 55, 427-438, 2006.

[50] B. Zhou, I. Gutman and T. Aleksić, A note on Laplacian energy of graphs, MATCH Commun. Math. Comput. Chem. 60, 441-446, 2008.

[51] B. Zhou and N. Trinajstić, On general sum-connectivity index, J. Math. Chem. 47, 210-218, 2010. 